\title{
The Development of Rapid Building 3D Scene Tool Based on Demands for Aging Adapting Functions in Old Residential Buildings
}

\author{
Xiaolei Zhao, a , Hong $\mathrm{Ji}^{1, \mathrm{~b}}$, Jianbing $\mathrm{Liu}^{2, \mathrm{c}}$ and Xinzhu Xing ${ }^{2, \mathrm{~d}}$ \\ ${ }^{1}$ Beijing Computing Center, Beijing 100094, China; \\ ${ }^{2}$ Beijing Research Center of Urban System Engineering, Beijing 100000, China. \\ azhaoxl@bcc.ac.cn, bjihong@bcc.ac.cn, ${ }^{\mathrm{c}}$ liujianbing@126.com, ${ }^{\mathrm{d}}$ xingxinzhu2008@126.com
}

Keywords: Rapid building 3D scene tool, unity3d, aging adapting, virtual reality.

\begin{abstract}
As the problem of aging of population becomes more and more obvious in China, Demands for aging adapting functions in existed residential buildings becomes much more urgent. Virtual Reality as a visual method has received a lot of attention. Using virtual reality method in existed residential buildings renovation will give great help to satisfy demands of aging adapting functions. According to the current situation of our country, in this paper, one rapid building three-dimensional (3D) scene tool based on virtual reality has been developed to meet the demands for aging adapting functions in existed residential buildings.
\end{abstract}

\section{Introduction}

As our society stepped into aging of population stage [1] and the government proposed "9073" senior retirement life planning [2], in which 97\% senior people live out their life in retirement in communities, community environment has become a very important part to senior people. Due to long-time implementation of one-child policy, a large amount of elderly people lack care from their children. Furthermore, elderly people usually centralize in old communities with insufficient facilities for the elders. So old communities' renovation becomes necessary and urgent. In order to help senior people renovate old communities, we use the method of virtual reality [3] to improve the visibility of community renovation and make renovation much more conveniently. In this paper, we designed and realized one rapid building 3D scene tool to meet the demand for existed residential buildings renovation. This rapid building 3D scene tool is developed based on Unity3D.

\section{Feasibility Analysis}

\subsection{Demands for aging adapting functions in renovation of old communities.}

Now the situation of China on aging population respect is that there are many empty nest families and people get old before become rich. Old communities play an important role in providing for the aged. So the old community's renovation is necessary and urgent. We should make effort to improve senior people's living condition.

\subsection{Modeling Technique of 3D Models.}

3D model is the base of virtual reality [4]. Because of Unity3D have no function of modeling, we model 3D models by means of 3D MAX. We model standard models that meet aging adapting requirements. In order to improve tool's performance, when modeling, we reduce unnecessary vertices, triangles and minimal size of textures.

@The paper supported by Government Special Fund in 2016 (PXM2016_178215_000006 and PXM2016_178215_000011) , Which name is" Development of one low cost and rapid building 3D scene platform based on demands for aging adapting functions in old communities" 


\subsection{Application base on Unity3d in Aspect of Virtual Reality.}

Though Unity3D is always used as one kind of game development software, Unity3D have successfully applied in many cases of virtual reality till now. H.J. Zhu realized virtual roaming system based on Unity3D [5]. S. Wang et al. treat Unity3D as the development platform for virtual reality [6].

\section{Overall Design of System}

Fig. 1 Overall

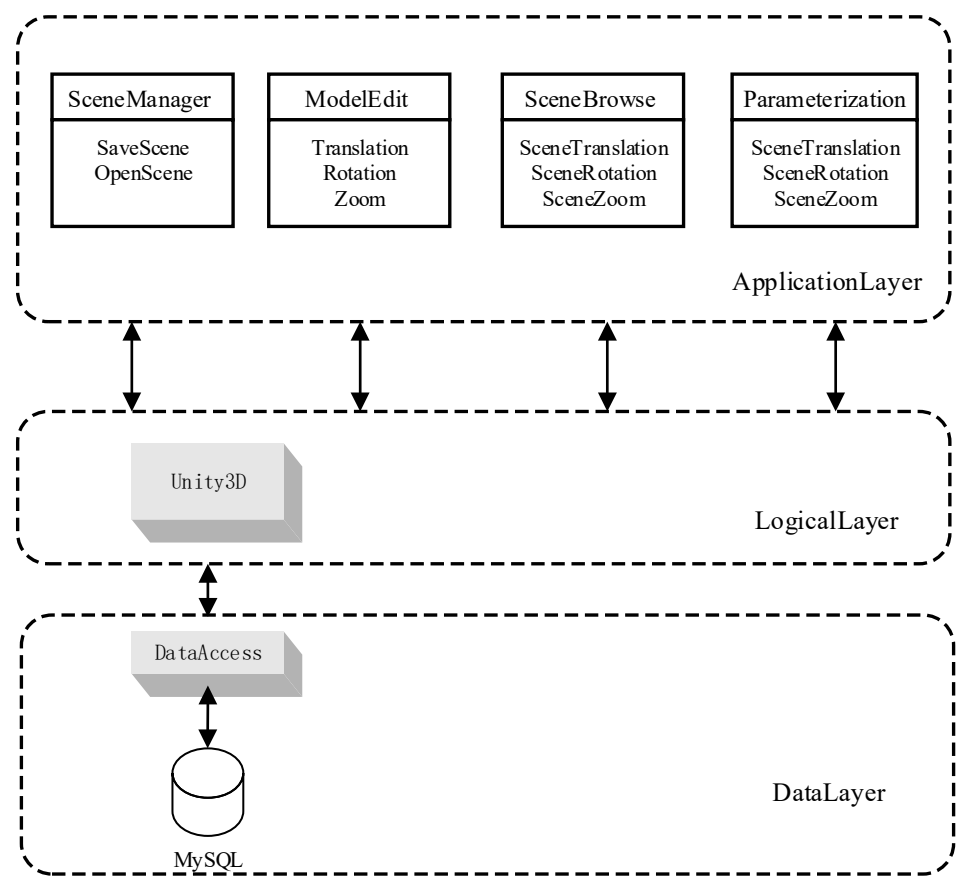

architecture

system of

\subsection{Overall System Architecture}

Our tool is a $\mathrm{C} / \mathrm{S}$ structure system. This tool run on windows platform which need windows 7 or higher than windows 7 version. The server what we used is Apache and the database is MySQL. Because we just need download models, so we adopt non-real-time interaction with the server.

We put all of the standard models, which are packed into resource files, on the server side. Users select models on the basis of their need, models will be download real-time. Then users can edit their models in scene. Fig. 1 describes the overall system architecture.

\subsection{Functions Overview of All the Modules}

1) Interaction with the Server: Because of there are lots of standard models of aging adapting, so we store models on the server side. Users select models saved on the server side to the scene by clicking and selecting specified category, specified button below the list. Fig. 2 shows the basic toolbar.

2) Model Editor: The model editor module is designed to achieve rapid arranging models and editing models. The user can move, rotate and zoom selected model by using corresponding tools. If users want to undo the last operation, they can achieve it by using the shortcut key Z. If they want to redo their last operation, they just need to press the shortcut key R. Users also could modify selected model's size by inputting directly. Fig. 3 shows the movement, rotation and zoom tool. Fig. 4 shows the panel which is used to directly modify model's size.

3) Scene Manager: Scenes created are much more meaningful if they can be saved, so our tool developed scene manager module. Users can save and open their scenes conveniently by selecting the save scene button and the open scene button.

4) Scene Browse/Camera Controller: To make it easy for users to build and browse scenes, our tool realized camera controlling functions. Users can move, rotate, and zoom angle of view by way of operating the keyboard and the mouse. 
5) Parameterization: This tool support change color and texture function to models which are only have one kind of material or which are specified one kind of material. In addition, users can control on-off and brightness of light component of models, which belongs to the light class. Fig. 7 shows the changing color and texture panel. Fig. 5 is the controlling light panel.

6) Else Functions:

a) Highlight: In order to that users selecting models conveniently, the model will display by bright color to remind the user when the mouse touch the model in the scene.

b) Model Search by name: This tool provides simple search function by means of inputting words which included in model's name. Fig. 6 shows the search function.

c) Replication and Delete: Using this tool also could copy and delete selected models according to users' need.

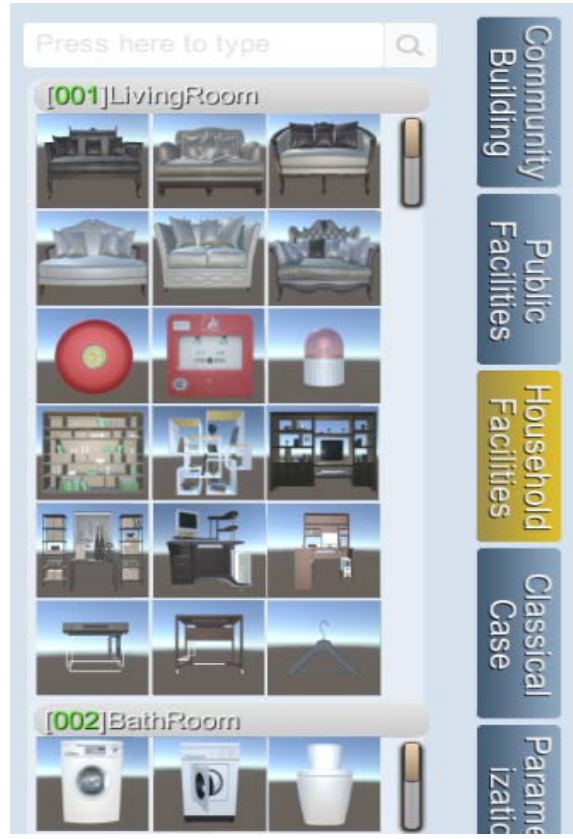

Fig. 2 Toolbar

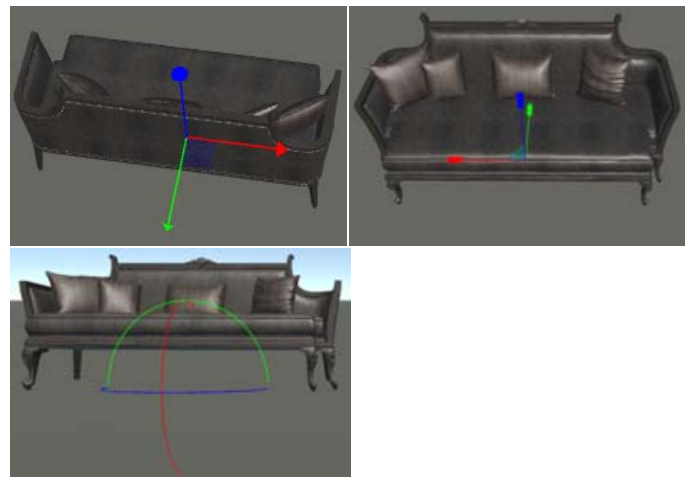

Fig. 3 Editing tool

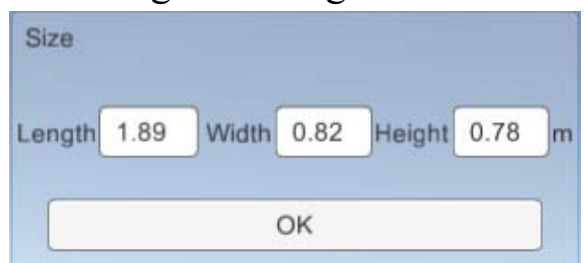

Fig. 4 Size editing tool

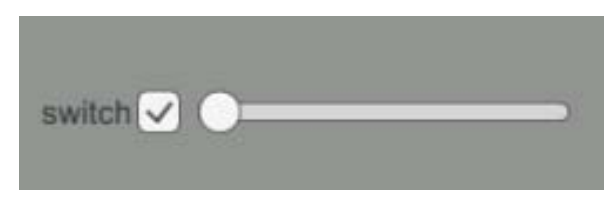

Fig. 5 Light tool

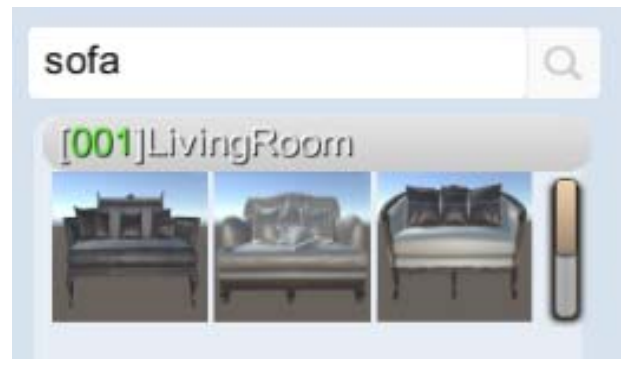

Fig. 6 Search pane1

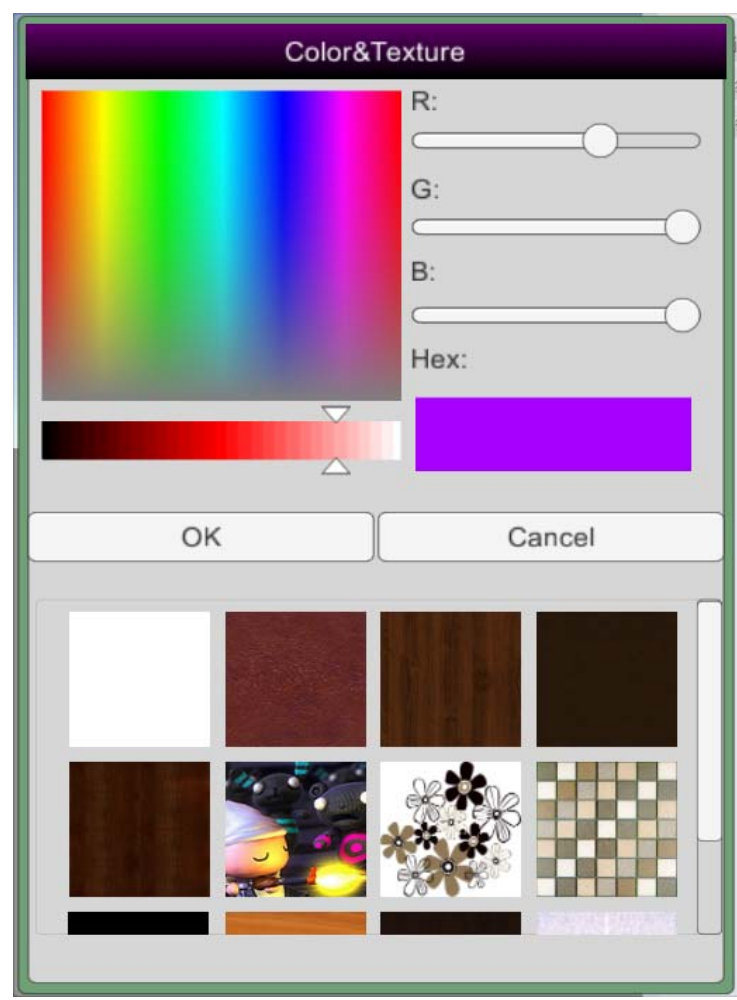

Fig. 7 Color and texture tool 


\section{Key Technologies and Implementations of the Functions}

\subsection{Interaction with the Server.}

According to the design scheme of the rapid building 3D scene tool, the user can select any models from toolbar and drag it into the scene. Unity3d interacts with web server by WWW class non-real-time. WWW class is based on HTTP protocol to achieve network transmission function. When the user selects one model at the client side, WWW class requests data by way of GET request. The server executed PHP scripts, search data from MySQL. If requested data exists, the server will return the address of the resource file. The client will proceed to download and parse operation. The resource file will be parsed into GameObject, which is the object in Unity3d. Then, the object could move along the ground follow the mouse moving.

\subsection{Model Editor Realization.}

We draw the editing tool real-time base on GL graphic library. GL graphic library is underlying graphic library, it can be used to draw 2D and 3D geometry [7]. When editing, we judge the position that is intersected by ray from camera to mouse position and coordinate planes belong to which coordinate planes of XY, XZ, YZ to confirm the user choose which axes. The selected axis become yellow when user's mouse hit this coordinate axes.

1) Movement: the user drag any coordinate axes, the selected model will move along that axis.

2) Rotation: the user drag any coordinate circle, the selected model will rotate along that axis.

3) Zoom: the user drag any coordinate axis, the selected model will zoom in or zoom out at the direction of the axis. Also the user could zoom the model on the whole.

This tool also supplies modifying size of models directly: Obtaining bounding box of one model and its child comes to modify model's size directly. Getting bounding box means getting model's length, width and height value.

In order to develop undo and redo functions, we store model's position value, rotation value and scaling value in the list data structure. When the user perform undo or redo operation, we assign stored value to model's property.

\subsection{Scene Manager Realization.}

Saving a scene actually is saving the model's position, rotation and scale information. So we save these information in XML files. When the user want to open one saved scene, indeed, this rapid building 3D scene tool reads the corresponding XML file and parse it, then download and replace these models. In this part, we call for Windows Forms dynamic link library file to use the open file dialog box function and the save file dialog box function. Fig. 8 show the xml structure of one scene file.

\subsection{Scene Browse/Camera Controller.}

We developed scene camera's movement, rotation and scaling functions to make building and browse easy. Operating model rely on mouse and keyboard input.

1) Camera zoom in or zoom out: Judging movement value in $Y$ axis of long-pressing mouse middle key come to modify camera's $Z$ axis value.

2) Camera Rotate: Judging movement value in $Y$ axis and $X$ axis of long-pressing mouse middle key come to modify camera's rotation angle.

3) Camera movement: Judging movement value in $Y$ axis and $X$ axis of long-pressing mouse middle key come to modify $\mathrm{X}$ axis and $\mathrm{Y}$ axis value of camera.

\subsection{Parameterization.}

In order to reach goal of high efficiency, we should make models recycled as much as possible. So we make part of models' appearance could be modified according to users' personal interest.

In this tool, we could change color value of the model, which only have one kind of material or is assigned one kind of material. Selected color from the color panel, just like Fig. 7, gives to the color property of the model's material. Similarly, we also could change model's texture value. Regarding the demand for adjusting light intensity, we just need to get model's light component for controlling on-off of light and adjust light component's value. 


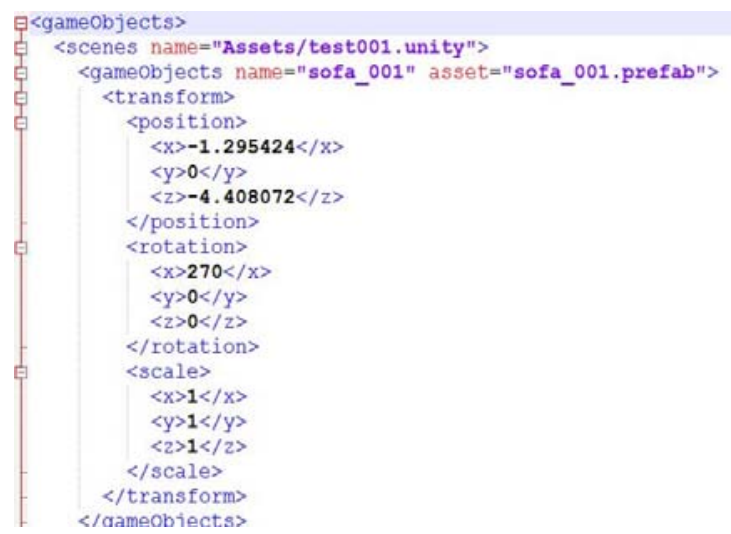

Fig. $8 \mathrm{Xml}$ file

\subsection{Basic Functions Realization.}

1) The Model Highlight: We develop highlight effect by judging collision between the mouse and the model which is detected by emitting ray and real-time drawing based on GL graphic library, the highlight material and mesh files of the model and its children.

2) The Model Search by name: We develop search function by matching input text and model's name. At the same time, we could control the toolbar dynamic display synchronously.

3) User Interface (UI) Development: UI is an important part of one system, it decides user's experience. Most popular UI system of Unity is UGUI and NGUI. UGUI and NUGI are all used in our tool now. To UI development, what we need to do is make UI components could response user's operation. In this part we mainly used delegate and events design mode to make UI work.

4) Performance optimization: In aspect of renderer, we make use of level of detail technology, make materials are recycled as much as possible and use optimized models, and so on. In aspect of CPU optimization, we put logic code out of update function as much as possible, load resources according to requirement, and so on.

\section{Summary}

Our country's current situation decides that old communities' renovation is necessary and urgent. Virtual reality could help old communities' renovation reduce costs and become visualization.

The rapid building 3D scene tool could let users build scenes what they image and could save scenes they build. This tool supplies sufficient standard models for aging adapting, including public and household facilities and so on. Also, this tool supplies the editing tool and the basic parameterized tool to users. In addition, what I have to say is that this tool comes from the demand for aging adapting in old communities but not only limiting in this area. This tool also could be used at apartment design area, and so on. At last, the rapid building 3D scene tool must become much more perfect and humanized in the future.

\section{Acknowledgement}

This work supported by Beijing Research Center of Urban System Engineering and Beijing Computing Center. Our models which reach the requirement of aging adapting functions are built based on data comes from Beijing Research Center of Urban System Engineering.

\section{References}

[1] Y.J. Jing, R.J. Chen, The development and management innovation of home care system in China: A perspective of coordination, Fudan Journal (Social Sciences Edition). 0 (2009) 133-140.

[2] Y.M. Zhou, J.Y. Lin, Analysis on the development and planning principles of senior living community, City Planning Review. 36 (2012) 46-51. 
[3] J. Steuer, Defining Virtual Reality: Dimensions Determining Telepresence, Journal of Communication. 42 (1992) 73-93.

[4] C.G. Li, F.Q. Zhu, L. Tan, C. Yang, Research on development approaches of virtual experiment based on 3D and virtools technologies, Computer Engineering \& Applications. 42 (2006) 84-86.

[5] H.J. Zhu, Virtual roaming system based on unity3d, Computer Systems \& Applications. 21 (2012) 36-39.

[6] S. Wang, Z.L. Mao, C.H. Zeng, H.L. Gong, S.S. Li, B.B. Chen, A new method of virtual reality based on unity3d, 2010 18th International Conference on Geoinformatics. 2010.

[7] Y.S. Xuan, Unity3D game development, Post\& Telecom Press, Beijing, 2012. 